\title{
Variación espacio-temporal de la respuesta espectral en manglares de La Habana, Cuba, evaluada con sensores remotos
}

\author{
Dennis Denis Ávila ${ }^{1 *}$, Emerio A. Curbelo ${ }^{1}$, Luis J. Madrigal-Roca ${ }^{1}$ \& Ramón D. Pérez-Lanyau ${ }^{1}$ \\ 1. Dpto. Biología Animal y Humana, Facultad de Biología, Universidad de La Habana, Cuba, calle 25 entre J e I, plaza, \\ La Habana, Cuba; dda@fbio.uh.cu, eacurbelo@fbio.uh.cu, ljmadrigal@estudiantes.fbio.uh.cu, \\ ramondperez@estudiantes.fbio.uh.cu \\ * Correspondencia
}

Recibido 27-IX-2019. Corregido 12-XII-2019. Aceptado 31-I-2020.

\begin{abstract}
Spatio-temporal variations of the spectral response in mangroves of Havana, Cuba, by remote sensing. Introduction: Mangroves are one of the most important forest types because of their ecosystem services and ecological roles. They represent $5 \%$ of the emerged land of Cuba. No previous studies are describing spectral vegetation indexes variations by remote sensing in Cuban mangroves, but these variables can be used as indicators of the conservation status of the ecosystem and sustain national wide assessments. Objective: in the current paper we describe spatial and temporal variations in two spectral vegetation indexes in four nearcity mangroves at Havana, Cuba and compare them to a natural control site. Methods: Study was conducted in localities named: Bajo de Santa Ana, Cojímar, Rincón de Guanabo, and El Cobre - Itabo lagoon and as control site was selected northern Zapata swamp. By using Climate Engine platform we extract, from 2460 Landsat satellite images, mean values of spectral indexes NDVI and EVI in 5 parcels per locality, from 1984 to 2019. Variables were statistically compared among localities and the global trend was described. Results: We detect geographic differences in both indexes, which can be related to structural properties and conservation degree of mangroves in each locality. Global trend of indexes was to increase, but differently among localities. Slighter changes appear in the control site and, among near city mangroves, in Rincón de Guanabo and Cojímar. The ordering of localities from spectral variables was consistent with the ranking in general conservation degree. Conclusions: Spectral responses describe uniquely each mangrove forest, in concordance to each ecological and conservation characteristics. There is a need for promoting studies using remote sensors at these forest types and to generate strong and reliable indicators that can sustain future researches and monitoring schemes in Caribbean mangroves.
\end{abstract}

Key words: coastal wetlands, NDVI, EVI, ecological indicators, historical trends.

Denis, D., Curbelo, E.A., Madrigal-Roca, L.J., \& Pérez-Lanyau, R.D. (2020). Variación espaciotemporal de la respuesta espectral en manglares de La Habana, Cuba, a través de sensores remotos. Revista de Biología Tropical, 68(1), 321-335.

Los manglares tropicales son bosques perennes halófitos que típicamente habitan las zonas costeras bajas y fangosas en las regiones tropicales. Se consideran entre los ecosistemas arbóreos más importantes desde el punto de vista ecológico y, a su vez, están entre los más amenazados (Ellison \& Farnsworth, 1996; Valiela, Bowen \& York, 2001; Alongi, 2002;
Gilman, Ellison, Duke \& Field, 2008). Aunque existen más de 50 especies de mangles, cada bosque está generalmente dominado por muy pocas especies, similares entre ellas y carecen de estratos inferiores de vegetación. A pesar de esto, los bosques de mangle tienen una elevada productividad primaria y mantienen extensas tramas alimenticias, tanto terrestres 
como marinas (Krauss et al., 2008; Nagelkerken et al., 2008). Los manglares contribuyen significativamente al mantenimiento de los recursos pesqueros de las áreas adyacentes y protegen las costas de la erosión por efecto del oleaje. Además, capturan y almacenan grandes volúmenes de carbono atmosférico, con efectos ambientales a nivel global.

En Cuba se estiman alrededor de 450000 ha de manglares, lo cual la ubica entre los primeros países del mundo en cuanto a extensión relativa de estos ecosistemas. Los mangles representan el $11 \%$ del área boscosa (Roman, 2018) y se ha sugerido que, de forma general, presentan un alto grado de conservación (Galford et al. 2018). Sin embargo, no están exentos de serias amenazas como el represamiento de los cauces de agua dulce para propósitos agrícolas, la contaminación y el desarrollo del turismo en la zona costera (Galford et al. 2018). Varias de las ciudades costeras más importantes del país se han desarrollado en sitios donde previamente existieron zonas de manglares, y estas han quedado reducidas a pequeños fragmentos embebidos en matrices de usos antropogénicos del suelo. En estos contextos, los tensores sobre el ecosistema aumentan a la par de su importancia ecológica. Pocos estudios se han realizado en estos manglares asociados a importantes núcleos urbanos, por lo que aún el conocimiento es insuficiente para lograr un manejo conservacionista eficiente.

Las técnicas de sensores remotos se emplean regularmente en la actualidad para el estudio y monitoreo de manglares (Green, Clark, Mumby, Edwards \& Ellis, 1998; Al Habshi, Youssef, Aizpuru \& Blasco, 2007; Giri et al., 2011, Heumann, 2011; Thomas et al. 2018), pero su empleo ha sido muy limitado en Cuba. Los métodos más generalizados para el estudio y monitoreo de los manglares cubanos se han enfocado más al empleo de datos de campo (Menéndez, Guzmán \& Capote, 2002; Guzmán \& Menéndez, 2013), aunque se reconoce la meta del uso de las imágenes satelitales. En el área del Caribe, estudios de esta naturaleza han sido desarrollados por autores como Jensen et al. (1991), Wang, Sousa, Gong
\& Biging (2004) y Everitt, Yang, Summy, Judd \& Davis (2007).

Dada la escasez de información precisa sobre los manglares de La Habana, su estado actual y sus tendencias históricas, el objetivo del presente trabajo es evaluar la variabilidad espacio temporal en dos índices espectrales de vegetación en cuatro manglares periurbanos de La Habana, Cuba. Este trabajo y el método empleado puede servir de referencia para su aplicación en otras localidades o formaciones vegetales, y los resultados pueden establecer una línea base para continuar el monitoreo de los manglares estudiados, que están sometidos continuamente a numerosos factores antropogénicos estresantes.

\section{MATERIALES Y MÉTODOS}

El estudio se desarrolló en cuatro localidades de La Habana, donde se encuentran los manglares más importantes: Bajo de Santa Ana, la laguna de El Cobre - Itabo, la desembocadura del río Cojímar y el Rincón de Guanabo. Estas localidades aparecen descritas en Denis et al. (Denis et al., en prensa) así como en la información suplementaria (https://doi. org $/ 10.6084 / \mathrm{m} 9$.figshare.9899132). Se incluyó, además, solo como referencia o control, cinco parcelas de mangles de la ciénaga de Zapata, que fue estudiada por Cissell \& Steinberg (2018) quiénes encontraron una alta estabilidad en su cobertura vegetal en los últimos 40 años. Bajo de Santa Ana está situado al oeste de la ciudad, en una llanura marina acumulativa, rodeada de terrazas costeras de origen calcáreo, y colindan directamente con zonas urbanas que drenan al mismo las aguas albañales. Su manglar está conformado por mangle rojo (Rhizophora mangle) (10\%) hacia el borde del mar, mangle prieto (Avicennia germinans) (45\%) y patabán (Laguncularia racemosa) (45 \%) según evaluaron Ventura et al. (2017). La laguna de El Cobre-Itabo, a $23 \mathrm{~km}$ al Este de la bahía de La Habana, se ha considerado el manglar mejor conservado y de mayor extensión del litoral norte de La Habana (Suárez, 2011), pero contiene un hotel ubicado dentro de su 
extensión e históricamente ha sido afectado por varios efectos antropogénicos. Este manglar está formado por extensas zonas de L. racemosa $(50 \%)$, bordeados de $R$. mangle (30\%) y A. germinans (10\%), con individuos dispersos de Conocarpus erectus (Yana) (3\%) (Ventura et al. 2017). El Rincón de Guanabo se ubica en el extremo oriental del sector Playas del Este, en un área declarada Paisaje Natural Protegido, precisamente por el desarrollo de sus manglares y la alta biodiversidad que sustentaba. Históricamente, estuvo formado por las cuatro especies de mangles, pero en la actualidad solo predomina $L$. racemosa hacia la zona marina y C. erectus hacia la zona interna. Y finalmente, el bosque de mangle de la desembocadura del río Cojímar se encuentra enclavado en el área del primer meandro de este río, antes de su desembocadura, en el poblado del mismo nombre. Con un área aproximada de siete hectáreas, se encuentra fisionómicamente bien conservado, al estar relativamente protegido por la restricción de acceso que supone la cooperativa pesquera ubicada en la desembocadura. Sin embargo, es directamente afectado por la intensa contaminación que arrastra el río. Está formado mayormente por $R$. mangle en las vertientes del río y $A$. germinans hacia el interior.

Para la descripción de la variabilidad espacial de los índices espectrales se empleó la plataforma Climate engine (Climate Engine 2019), que permite el acceso rápido a información de sensores remotos (Huntington et al., 2017). Se utilizaron los índices espectrales calculados a partir de 2460 imágenes satelitales de la serie Landsat (4/5/7/8) entre mayo de 1984 y mayo de 2019 (35 años). Para el análisis espacio temporal se extrajeron dos índices principales: el Índice de Diferencia Normalizada de Vegetación (NDVI) y el Índice de Vegetación Mejorado (EVI), que son los índices más ampliamente empleados en el monitoreo de la vegetación por asociarse a numerosos aspectos ecológicos y funcionales de la misma. Sin embargo, para una mejor caracterización espectral y ordenamiento de las localidades se incluyeron en los análisis globales multidimensionales el Índice de Diferencia Normalizada de Agua (NDWI) y la Tasa Azul-Verde (BG). Estos otros índices reflejan otros aspectos del follaje de las plantas, como el estado hídrico o la composición de pigmentos fotosintéticos y pueden complementar al NDVI y EVI en la descripción de la vegetación. En la información suplementaria se puede acceder a las fórmulas, fuentes y descripciones estadísticas generales de estos otros dos índices (https://doi. org $/ 10.6084 / \mathrm{m} 9$.figshare.9899132).

Para caracterizar espectralmente cada manglar se tomaron los valores promedios mensuales en cinco polígonos de cada localidad con áreas entre 100 y $250 \mathrm{~m}^{2}$ en dependencia de su extensión, durante todo el periodo de estudio. Las parcelas estuvieron a distancias mayores de $60 \mathrm{~m}$ entre ellas. Las muestras totales (parcelasimagen) estuvieron distribuidas de la siguiente forma: 2128 registros en Bajo de Santa Ana, 1892 en Cojímar, 1671 en Guanabo, 2435 en El Cobre - Itabo y 3574 en la ciénaga de Zapata, para un total de 11700 muestras.

Las tendencias centrales de los datos se describieron usando la mediana y para describir la variabilidad se calcularon los cuartiles, excepto en el análisis de las variaciones anuales donde se empleó la media y los límites de confianza al $95 \%$. Para suavizar la extrema variabilidad en los datos, en los análisis de las tendencias históricas se utilizó una media móvil de ventana 3 y para su análisis estadístico se promediaron los valores por meses. Se compararon los índices espectrales entre las áreas en la actualidad (año 2019) y se describió la tendencia histórica de cada una, por medio de la pendiente global de la línea de tendencia de cada índice y comparando los valores medios de los primeros y últimos seis años del intervalo de estudio. Se calcularon las variaciones estacionales generales (asumiendo dos estaciones climáticas: estación de seca (invierno) (de enero a abril y de septiembre a diciembre) y estación de lluvias (verano) (de junio hasta agosto) y su comportamiento anual, de manera general, para todos los manglares estudiados y se comparó con el control. Para controlar los niveles de redundancia en la información en los índices espectrales y representar las localidades 
en un espacio de ordenamiento multivariado se realizó un Análisis de Componentes Principales. Todo el análisis de los datos se hizo en el programa Statistica v8.0 (StatSoft, 2007).

\section{RESULTADOS}

Los índices espectrales reflejan las características de la cobertura vegetal de los manglares, que a su vez se relaciona con su grado de conservación y las características ambientales de los lugares. Cómo cada índice se correlaciona específicamente con distintos parámetros y tienen diferentes propiedades estadísticas, su comportamiento entre manglares fue diferente (Fig. 1) en el momento actual (mayo - junio, 2019). Bajo de Santa Ana junto a los manglares de El Cobre - Itabo, mostraron los mayores valores de NDVI, incluso superando al sitio control, en la Ciénaga de Zapata. En el índice EVI, también Bajo de Santa Ana superó al sitio control, pero este último supera al resto de los manglares periurbanos de La Habana. Este manglar, en el extremo occidental de la ciudad tuvo los índices mayores, excepto en la tasa BG. El manglar de El Cobre - Itabo presentó
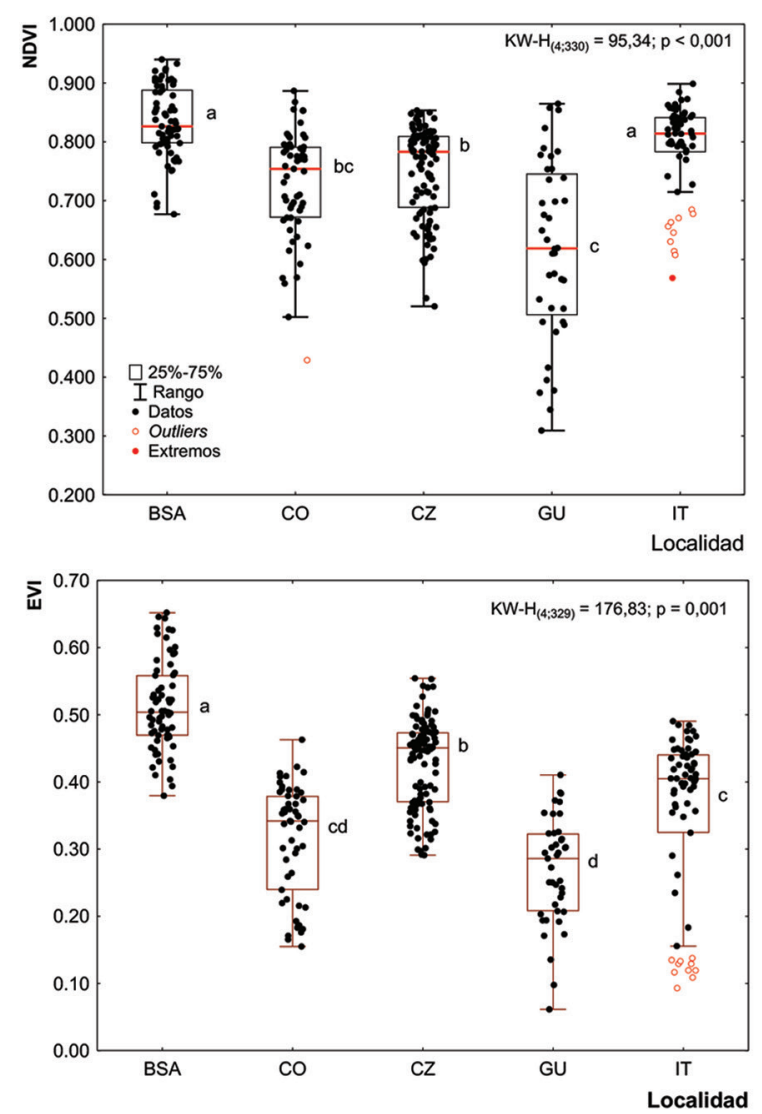

Fig. 1. Comparación de los valores promedios de los índices espectrales NDVI y EVI en manglares periurbanos de la ciudad de La Habana, Cuba, y un sitio natural de control (Ciénaga de Zapata) en mayo - junio 2019. Localidades: BSA: Bajo de Santa Ana, IT: El Cobre-Itabo, CO: Cojímar, GU: Guanabo y CZ: Ciénaga de Zapata. Letras diferentes indican diferencias estadísticas según la prueba de rangos a posteriori.

Fig. 1. Comparison among mean values of NDVI and EVI spectral indexes in mangroves near Havana city, Cuba, and a control natural site (at Zapata Swamp) in May - July, 2019. Localities: BSA: Bajo de Santa Ana, IT: El Cobre-Itabo, CO: Cojímar, GU: Guanabo y CZ: Zapata Swamp. Different letters indicate statistical differences according to a posteriori rank test. 
mayor valor de EVI que el de Guanabo, mientras que los mangles de la desembocadura del río Cojímar tuvieron valores intermedios entre ambos. Los manglares del Rincón de Guanabo y de la desembocadura del río Cojímar tuvieron los menores índices, generalmente similares entre ellos aunque con una ligera tendencia a mayores valores en Cojímar.

La tasa BG fue el índice espectral que mostró menos diferencias entre manglares, con una tendencia gradual a disminuir en sus valores centrales desde El Cobre - Itabo, localidad que mostró una dispersión en este índice muy superior al resto de los manglares, hasta Guanabo. Los valores, sin embargo se superponían marcadamente. De forma general, todos los índices mostraron altas variabilidades, con coeficientes de variación desde un $16.5 \%$ en el NDVI, hasta $39.9 \%$ en la tasa BG. Los índices EVI y NDWI tuvieron variabilidades de 24 y $27 \%$, respectivamente.

De forma general, todos los índices mostraron ligeras variaciones anuales en sus tendencias centrales, que no coincidieron exactamente con los meses de las estaciones climáticas tradicionalmente establecidas en Cuba, sino que disminuían al inicio del año y tendían a aumentar durante la temporada de lluvias. Las tendencias anuales no son consistentes por igual entre todos los manglares estudiados, sino que cambios estacionales marcados solo aparecen en algunas localidades (Fig. 2). El NDVI mostró cambios estacionales solo en los manglares de Cojímar, Bajo de Santa Ana y en el sitio
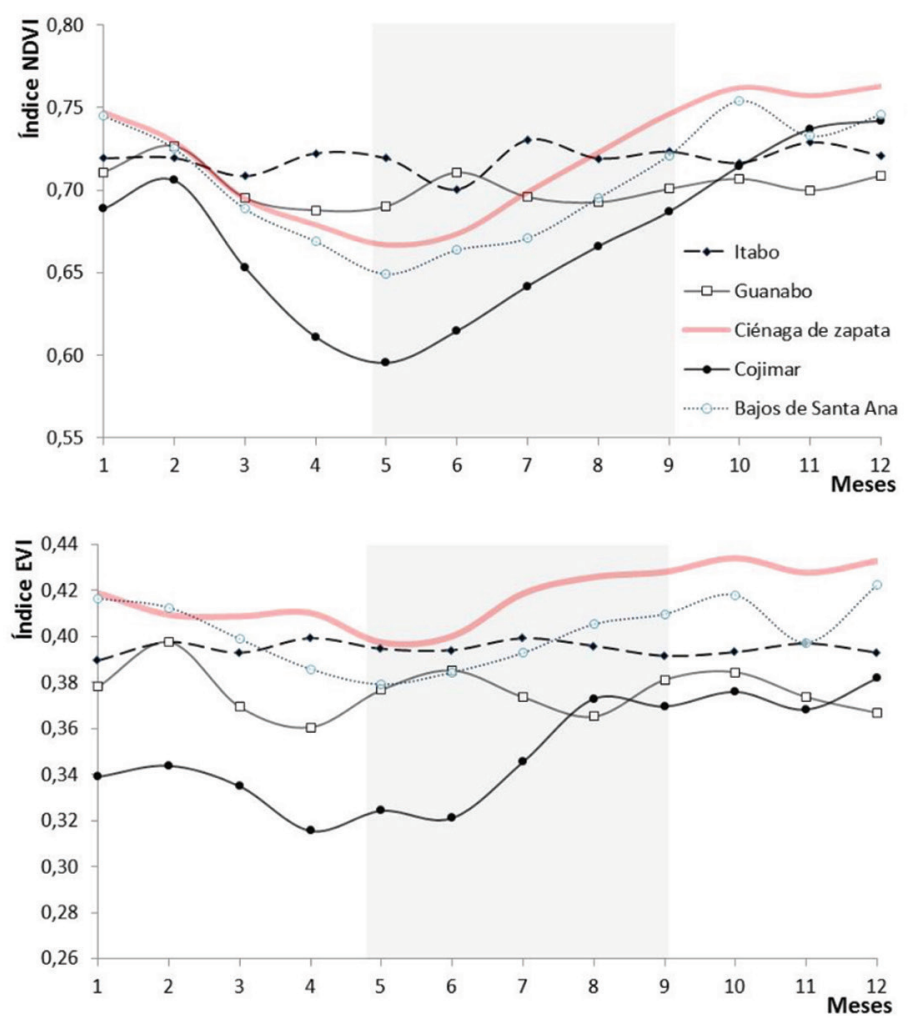

Fig. 2. Variación anual de los índices espectrales de vegetación NDVI y EVI en manglares periurbanos de la ciudad de La Habana, Cuba, y un sitio natural de control (Ciénaga de Zapata) (valores mensuales promedio entre 1984 - 2019). En gris se marca el intervalo de meses considerados como la estación de lluvias.

Fig. 2. Annual variation in spectral vegetation indexes NDVI and EVI in mangroves near Havana City, Cuba, and in a natural control site (Zapata Swamp) (monthly values averaged between 1984 - 2019). The interval of months considered as the rainy season is marked in gray. 
control en la Ciénaga de Zapata, con tendencias similares al EVI. Los manglares de Cojímar fueron los que mostraron cambios estacionales más marcados en los índices, mientras que en la laguna El Cobre - Itabo y el Rincón de Guanabo no se evidenció estacionalidad en ningún índice espectral. En los manglares de El Cobre - Itabo los índices EVI y NDVI fueron notablemente constantes dentro del año.

Las tendencias históricas en el comportamiento de los índices espectrales, entre 1984 y 2019, se muestran en la Fig. 3 y en la Fig. 4. Las pendientes de las líneas de tendencia sugieren el sentido y magnitud de cambios de los índices globales durante el periodo de estudio. Bajo de Santa Ana muestra la tendencia al incremento histórico del NDVI y del EVI más marcados, seguida de Cojímar y El Cobre Itabo. El sitio control en la ciénaga de Zapata mostró solo una ligera tendencia al incremento. Excepto en Bajo de Santa Ana, el índice EVI mostró disminuciones marcadas en los últimos años, lo cual fue detectado con el NDVI solo para el Rincón de Guanabo.

A manera de resumen de los cambios históricos, se compararon los valores medios de cada índice entre los primeros seis años del periodo de estudio (1984 - 1990) y los últimos seis años (2013 - 2019) (Fig. 5). Entre ambos momentos, todos los índices tendieron a aumentar en mayor o menor grado, siendo el NDVI el que aumentó en mayor proporción y de manera más consistente en todas las localidades. Los menores cambios se produjeron en el sitio control y, entre los humedales periurbanos, en el Rincón de Guanabo y en Cojímar. Los mayores incrementos de NDVI y EVI se dieron en los Bajo de Santa Ana e Itabo.

Para caracterizar el comportamiento espectral de una manera más integral, se agruparon los índices de vegetación descritos (NDVI y EVI) a otros dos índices que tienen mayor sensibilidad a distintos factores ambientales, el índice normalizado de diferencia de agua (NDWI) y la tasa Azul - Verde (BG) en un análisis de componentes principales. Incluyendo solamente las muestras más actuales, este análisis permitió resumir el $87.2 \%$ de la variabilidad del sistema en los dos primeros ejes (Fig. 6) $(\mathrm{CP} 1=66.3$ y CP2 $=20.94)$ y se llegó al $97 \%$ con el tercero $(\mathrm{CP} 3=9.76)$. El ordenamiento de las parcelas en este espacio multidimensional evidenció distintas respuestas espectrales entre localidades, excepto en algunas parcelas en específico que se alejaban de la tendencia en cada sitio. El Rincón de Guanabo fue quien más se alejó del resto de las localidades según su respuesta espectral. El promedio de los puntajes en el primer componente siguió un ordenamiento perfecto desde el humedal mejor conservado (Bajo de Santa Ana) hasta el más afectado (Rincón de Guanabo), lo cual sugiere que este estadígrafo pudiera servir de indicador de grado de conservación de estas formaciones vegetales. En el primer componente, fueron el NDVI y el EVI los índices de mayor carga, mientras que en el segundo eje fue la Tasa BG.

\section{DISCUSIÓN}

Para poder desarrollar aplicaciones regionales o locales, relacionadas con la identificación y clasificación de características ambientales asociadas a la vegetación a partir de sensores remotos, se requiere del conocimiento de la estructura y función de la vegetación y sus propiedades reflectivas (Jensen et al. 1991; Heumann 2011). Esto incluye la comprensión de sus propiedades espectrales y respuestas en distintas partes del espectro electromagnético, por lo que es indispensable un conocimiento previo de la variabilidad espacial y temporal de los índices espectrales tradicionalmente empleados, en las diferentes formaciones vegetales. Este conocimiento permite establecer el vínculo entre las estructuras vegetativas y su condición, con el comportamiento reflectivo en un sistema ecológico de interés (Asner, 1998; Kuenzer, Bluemel, Gebhardt, Quoc \& Dech, 2011). En lugares como Cuba, donde no existen estudios previos, estas caracterizaciones básicas son necesarias como línea de base para estudios posteriores. Aunque existe una extensa tradición de estudios y monitoreo de manglares en Cuba, estos 


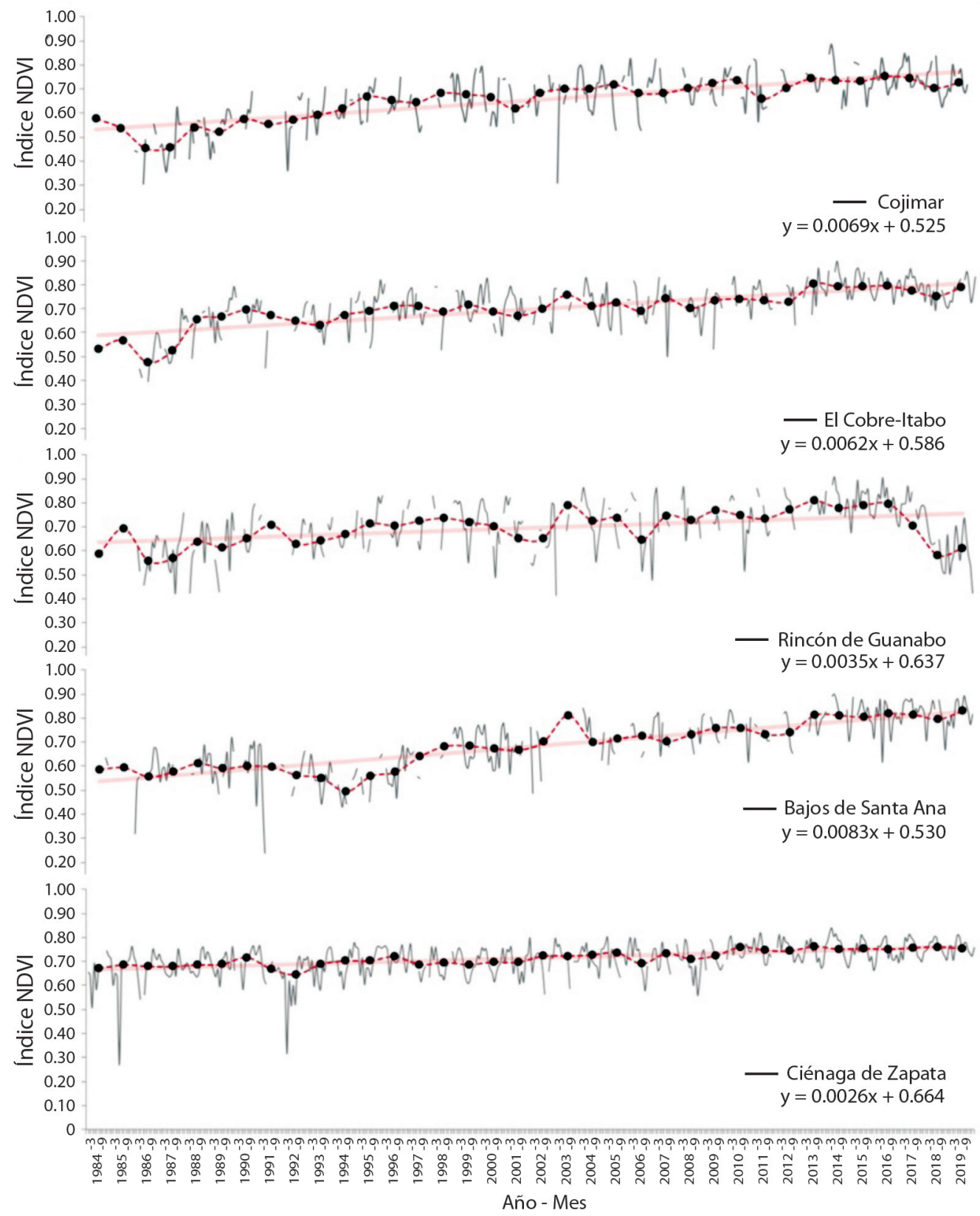

Fig. 3. Tendencias históricas en el Índice de Vegetación de Diferencia Normalizada (NDVI) de cuatro manglares periurbanos de la ciudad de La Habana, Cuba, y un sitio natural de control (Ciénaga de Zapata) entre mayo 1984 y junio 2019. La línea quebrada representa el comportamiento de los promedios mensuales de cinco parcelas en cada localidad; los puntos negros, las medias anuales y la línea continua, la tendencia global.

Fig. 3. Historical trend in Normalized Difference Vegetation Index (NDVI) in four near city mangroves at Havana, Cuba, and in a natural control site (Zapata Swamp) between May, 1984 and June, 2019. Broken line represent monthly average trend in five parcels of each locality; black dots mean annual averages and continuous line, the global trend. 


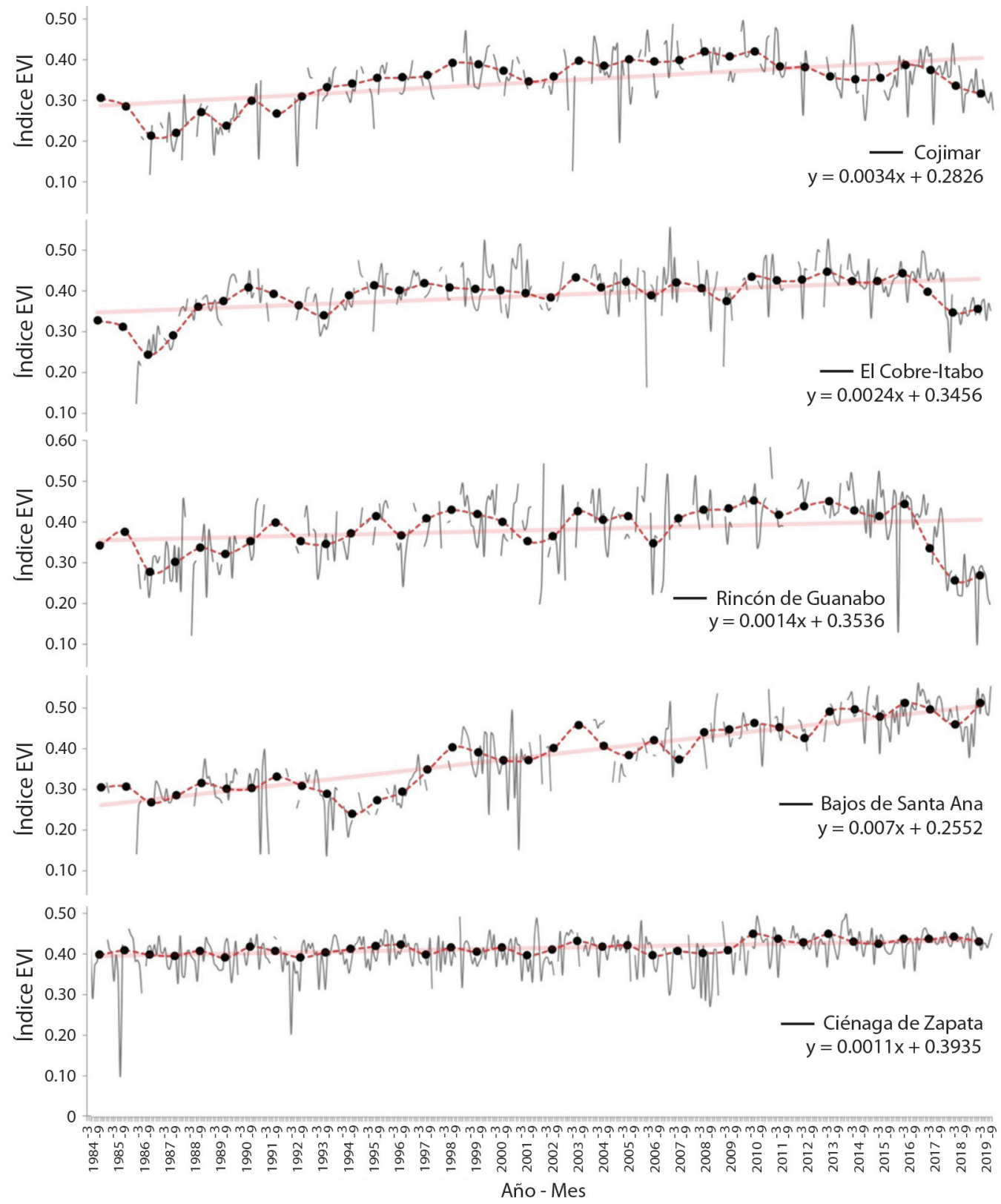

Fig. 4. Tendencias históricas en el Índice de vegetación Mejorada (EVI) de cuatro manglares periurbanos de la ciudad de La Habana, Cuba, y un sitio natural de control (Ciénaga de Zapata) entre mayo 1984 y junio 2019. La línea quebrada representa el comportamiento de los promedios mensuales de cinco parcelas en cada localidad; los puntos negros, las medias anuales y la línea continua, la tendencia global.

Fig. 4. Historical trend in Enhanced Vegetation Index (EVI) in four near city mangroves at Havana, Cuba, and in a natural control site (Zapata Swamp) between May, 1984 and June, 2019. Broken line represent monthly average trend in five parcels of each locality; black dots mean annual averages and continuous line, the global trend. 

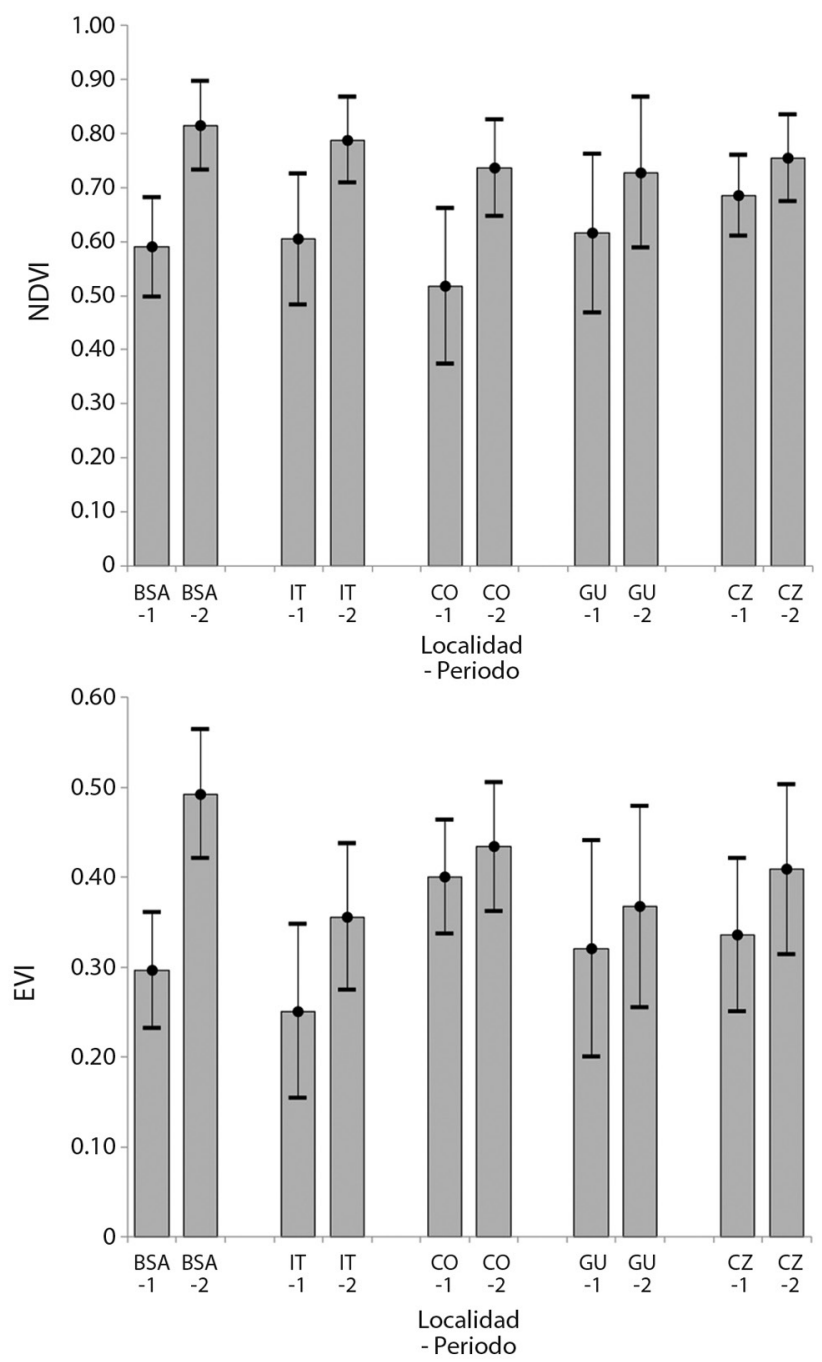

Fig. 5. Diferencias medias entre los índices espectrales de vegetación NDVI y EVI en cuatro manglares periurbanos de la ciudad de La Habana, Cuba, y un sitio natural de control (Ciénaga de Zapata) entre un periodo antiguo (1: 1984-1990) y la actualidad (2: 2011-2019). Localidades: BSA: Bajo de Santa Ana, IT: El Cobre-Itabo, CO: Cojímar, GU: Guanabo y CZ: Ciénaga de Zapata. Las líneas representan la desviación estándar.

Fig. 5. Mean differences among spectral vegetation indexes NDVI and EVI in four near city mangroves around Havana, and in a natural control site (Zapata Swamp) between the first years of the study (1: 1984-1990) and current years (2: 2011-2019). Localities: BSA: Bajo de Santa Ana, IT: El Cobre-Itabo, CO: Cojímar, GU: Guanabo and CZ: Zapata Swamp. Whiskers represent standard deviation.

se han centrado más al empleo de datos de campo (Menéndez \& Guzmán, 2006; Guzmán \& Menéndez, 2013), y aunque se ha reconocido como meta el uso de las imágenes satelitales, aún la cantidad de investigadores que dominan estas técnicas es insuficiente.
Los sensores remotos dan información para la detección de cambios y sirven de apoyo para la evaluación de los ecosistemas, especialmente su productividad, y en general son de gran ayuda para ganar una mayor comprensión de las relaciones ecológicas y biológicas, así como los procesos, funciones y dinámicas 


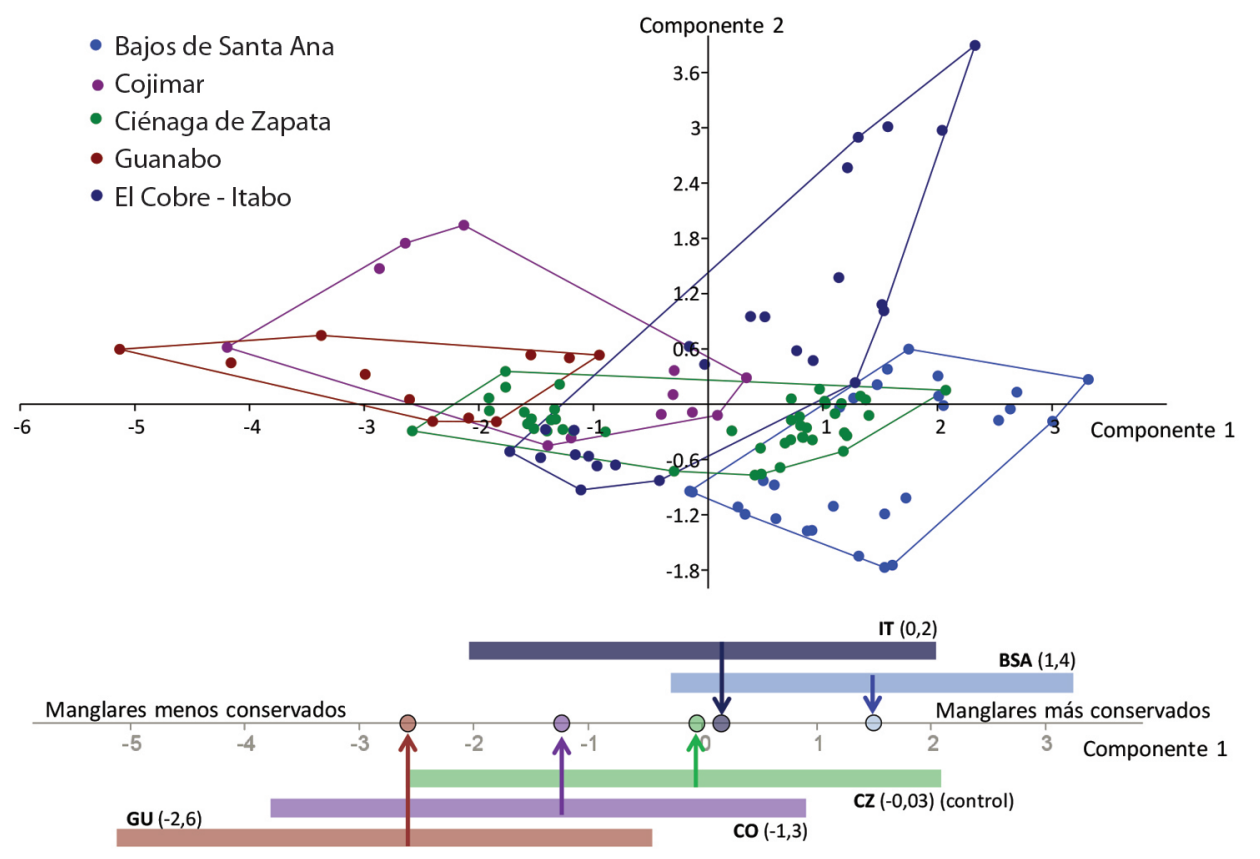

Fig. 6. Ordenamiento en el espacio de los dos primeros componentes principales obtenidos con cinco variables espectrales de los manglares en cuatro localidades de La Habana, Cuba, entre abril y julio 2019. Localidades: BSA: Bajo de Santa Ana, IT: El Cobre-Itabo, CO: Cojímar, GU: Guanabo y CZ: Ciénaga de Zapata.

Fig. 6. Ordering in two principal components obtained from five spectral indexes of mangroves at four localities of Havana, Cuba, between April and July in 2019. Localities: BSA: Bajo de Santa Ana, IT: El Cobre-Itabo, CO: Cojimar, GU: Guanabo and CZ: Cienaga de Zapata.

del ecosistema (Green et al., 1998, Tong et al., 2004, Vaiphasa, Skidmore \& Boer, 2005). Se ha comprobado que la información espectral también mejora grandemente los resultados de los estudios de mapificación o evaluaciones (Green et al., 1997; Kovacs, Wang \& BlancoCorrea, 2001). El empleo de índices espectrales, en lugar de las bandas originales de los sensores remotos mejora la información que se puede obtener de ellos (Xue, HaiShan \& Zhi Hua, 2011), y específicamente los índices espectrales de vegetación, resultantes de diferenciar, relativizar u ortogonalizar las reflectancias de distintas bandas de un sensor, pueden proveer medidas cuantitativas indicadoras de propiedades físicas de la cubierta vegetal, como el "verdor", la densidad de la canopia o el índice de área foliar (Green et al., 1997). Los índices que se obtienen por sensores remotos pueden además apoyar estudios de estimaciones de biomasa, productividad, secuestro de carbono, y otras variables globales (Heumann, 2011).

Decenas de índices de vegetación han sido descritos y formulados, cada uno con sus propiedades y correlaciones con variables biológicas, ecológicas o geofísicas. Muchas veces, a pesar de sus especificidades, se correlacionan también entre ellos, resultando en información redundante. Ello, unido a la alta variabilidad espacial y temporal relacionada tanto a los tipos de vegetación y condiciones del trasfondo (Huete, 1988) como a los efectos atmosféricos y climáticos, hace que no todos sean útiles en todos los contextos. Por todo esto, la selección de los índices espectrales específicos a utilizar en un estudio de vegetación es un aspecto complejo y puede ser necesaria la inclusión de otros índices más enfocados a otras condiciones ambientales (suelo, agua, temperatura) como 
complemento, como se hizo en este trabajo con el NDWI.

El NDVI tiene un rango desde -1 a 1 , aunque la vegetación verde naturalmente tiene entre 0.2 a 0.8 , pero ha sido criticado por el hecho de que se satura en condiciones de altas coberturas (lo que quiere decir que deje de detectar diferencias leves) y por ello se propuso el EVI. Se decidió incluirlo en este estudio porque continúa siendo un indicador de referencia y para condiciones de vegetaciones sin coberturas absolutas ha mostrado una amplia aplicabilidad. El índice EVI fue desarrollado como un producto estándar del MODIS para optimizar al NDVI en las regiones de alta cobertura vegetal (altos valores de LAI - Leaf Area Index) (Huete et al., 2002). Este índice usa la reflectancia en el azul para corregir la señal del sustrato y reducir las influencias atmosféricas (como la dispersión por aerosoles).

Los valores de los índices espectrales de vegetación fueron consistentes con las características macroestructurales de los manglares (densidad, altura, coberturas, etc.). Los manglares de Bajo de Santa Ana y de El Cobre-Itabo destacaron por los mayores valores mientras que el Rincón de Guanabo reflejó los menores, en concordancia con su mayor nivel de degradación. La capacidad de los índices espectrales de diferenciar los lugares, teniendo en cuenta sus diferencias en cuanto a los parámetros del manglar, apoyan su utilidad para las mapificaciones de diferencias relativas a gran escala.

Los manglares de El Cobre - Itabo y Bajo de Santa Ana mostraron las mejores condiciones estructurales y superaron en varios índices espectrales incluso al sitio control en la Ciénaga de Zapata. Esta última es el humedal más extenso del Caribe y sus manglares fueron estudiados con sensores remotos por Cissell \& Steinberg (2018), quienes encontraron una alta estabilidad en su cobertura vegetal en los pasados 40 años. Esto es consistente con que solo mostrara ligera tendencia al incremento histórico en los índices espectrales. En el caso de los manglares periurbanos estudiados, los efectos físicos destructores de varios ciclones recientes pueden haber influido en las disminuciones recientes en las respuestas espectrales, detectado de manera más precisa por el EVI (excepto en Bajo de Santa Ana), así como otras influencias antropogénicas. En visitas actuales a estos manglares se detectaron evidencias del efecto destructor de los vientos ciclónicos en todos ellos, excepto en Cojímar.

Las elevadas dunas de la costa de Itabo protegen al manglar contra el oleaje extremo pero la fragmentación impuesta por los viales y el efecto del hotel y sus descargas pueden representar amenazas potenciales a largo plazo a este ecosistema, considerado por Suárez (2011) como el manglar mejor conservado del litoral norte de La Habana. Según Menéndez, Guzmán \& Capote (2002), el manglar de Bajo de Santa Ana fue seriamente afectado por un dragado del río de Santa Ana en 1983, y su utilización como depósito de arena hasta el año 1989. Narran estos autores que al inicio de los años 90 en la región occidental el manglar había desaparecido totalmente, pero una década más tarde mostraría una notable recuperación natural. Aunque estos eventos mencionados no se reflejan en los datos históricos de los índices espectrales, es esta localidad la que mayores incrementos históricos de NDVI y EVI ha tenido.

Los manglares de Cojímar y del Rincón de Guanabo mostraron las respuestas espectrales más afectadas de las cuatro localidades. In situ, los manglares de Cojímar muestran evidencias de mayor impacto por la contaminación del río, pero desde el punto de vista estructural sus árboles no tienen las mismas afectaciones que en otras regiones. Ello se manifiesta en un mayor porte, diámetros de troncos y densidad del follaje, posiblemente relacionado a su ubicación geográfica al fondo de una bahía, que limita el efecto del oleaje extremo y en la cuenca de un valle, relativamente protegido de los vientos extremos. El resto de los manglares no cuenta con esta protección y por ello, ha sido más afectado por los ciclones recientes y estructuralmente, tienen diferentes características. La marcada estacionalidad de los índices espectrales en esta localidad, posiblemente se 
asocie a su composición de especies y al efecto de los cambios en el cauce del río.

Los manglares del Rincón de Guanabo, por otro lado, muestran las mayores afectaciones físicas en los años recientes con una degradación extrema en relación su estado histórico, producto del efecto de los ciclones, con intrusión de la duna costera, cambios hidrológicos que afectan el recambio de agua de la laguna interior y salinización progresiva de los suelos que resultan en cambios en la composición de especies y en una fuerte defoliación.

La vegetación responde de manera muy marcada a las variaciones climáticas, especialmente relacionadas con las precipitaciones. Sin embargo, estas respuestas son muy diferentes entre distintas formaciones vegetales. En el caso de los ecosistemas de humedales, donde el agua no suele ser limitante en ningún momento del año la respuesta a las precipitaciones puede ser menos evidente, lo que puede justificar que la estacionalidad marcada en los índices espectrales solo aparezca en algunas localidades.

A pesar de esto, muchos patrones fenológicos se han establecido evolutivamente en las especies a partir de tendencias temporales históricas del clima y, al estar genéticamente determinado, ello no cambia con facilidad. Es conocido que la respuesta espectral varía en relación al estado fenológico de la vegetación. En L. racemosa la floración comienza en abril y se extiende hasta octubre, mientras que los frutos son más abundantes a partir de junio (Menéndez, Guzmán \& Capote, 2002). Avicennia germinans tiene un período de floración concentrado entre mayo y junio, aunque pueden aparecer flores desde abril y hasta julio, mientras que los frutos o propágulos maduran de junio a agosto, aunque se han observado frutos hasta noviembre. Rhizophora mangle no presenta estaciones definidas de floración y fructificación, sin embargo, Pastor-Guzmán, Dasha \& Atkinson (2018) evaluaron y mapearon variables fenológicas en bosques de mangles a partir de series de tiempo de satélites de resolución media en el SE de México y concluyeron que era posible detectar cambios en la fenología de los mangles aun cuando no se había descrito previamente una estacionalidad marcada. Las diferencias reflectivas de las hojas entre las especies, aunque se han descrito empleando espectro-radiómetros in situ (Boon, Zubir, Jafri \& Hwee 2011), no deben influir en este caso ya que solo serían detectables con imágenes de mucha mayor resolución espectral.

Este efecto puede justificar la asincronía aparente entre los cambios de la respuesta espectral y los límites de las estaciones de seca y lluvia. También debe considerarse que los patrones de lluvias son cada vez más erráticos y los límites entre estaciones son cada vez más difusos producto de los cambios climáticos globales.

Sin embargo, si bien, estadísticamente se demuestra la existencia de una variación anual de los estadísticos de tendencia central de los índices de vegetación, debe tenerse mucho cuidado con la interpretación de esta variación, ya que la magnitud de los cambios es muy ligera: entre $0.66-0.74$ en NDVI ( $10.5 \%$ del rango de variación real de este índice) y entre 0.37 0.41 en EVI (6.5 \%). Aquí hay que considerar que estos cambios se refieren a tendencias centrales, que provienen de conjuntos de datos de muy alta variabilidad, con coeficientes de variación que alcanzan hasta casi un $40 \%$. No queda claro, si esta magnitud de cambio puede tener o no implicaciones ecológicas marcadas. Esto también apoya el empleo de imágenes de diferentes meses en estudios particulares, cuando no hay disponibilidad de información sincrónica, al ser muy probable que las diferencias entre meses queden enmascaradas en la variabilidad global y no sean detectables en estudios a corto plazo.

Este trabajo presenta, por primera vez, los valores de estos índices espectrales de vegetación para los manglares cubanos, sin embargo, las localidades estudiadas (exceptuando el control) no son necesariamente referentes en relación a la situación de los manglares a nivel nacional, por el efecto de la antropización impuesta por la cercanía a la ciudad más poblada del país. Por ello, la ampliación de este estudio a localidades menos impactadas es un paso 
obligatorio para poder estandarizar el empleo de los indicadores utilizados en este trabajo.

También debe recordarse que los índices de vegetación no están diseñados para cuantificar exactamente propiedades físicas absolutas de los componentes de la vegetación, sino para ser usados en la mapificación de cantidades relativas de estos componentes (Asner, 1998), que pueden entonces ser interpretados en términos de condiciones del ecosistema. Satyanarayana, Mohamad, Idris, Husain \& Dahdouh-Guebas (2011) muestran, a partir de una combinación de medidas en tierra y datos de sensores remotos, que se puede obtener información útil para la evaluación del estado los manglares en Malasia. En otras especies de plantas asociadas a manglares se ha demostrado que las influencias de la salinidad del suelo y la humedad pueden reflejarse en el comportamiento espectral (Hardisky, Gross \& Klemas, 1986). La combinación multidimensional de indicadores espectrales debe explorarse a más profundidad para encontrar maneras eficientes de monitorear el estado de estos ecosistemas.

En el presente estudio no se emplearon firmas espectrales de los mangles debido a que la respuesta espectral se caracterizó por medio de índices y no por las reflectancias nativas, además de que en las áreas estudiadas, la mezcla de individuos de especies es relativamente alta y no permite la separación de especies. La necesidad de datos hiperespectrales y de las firmas para separar las especies de mangles ha sido enfatizada por Thangaradjou, Sivakumar, Anantharaman \& Kannan (2007) y Shah \& Anjali (2007). Varios estudios se han enfocado en determinar las propiedades espectrales (en el rango visible y NIR) e identificar las longitudes de onda más importantes para la demarcación de las especies de mangles (Kamaruzaman \& Kasawani, 2007; Boon et al. 2011). En la India, un conjunto de características estándares de reflectancia fue establecida para discriminar las principales comunidades de manglares y sus especies (Nayak, Anjali, \& Shah, 2003), lo cual debiera ser un paso obligado en Cuba para sentar la base de un protocolo de monitoreo de mangles basado en sensores remotos.
Nuevas herramientas científicas de cómputos masivos online (on-cloud computing) asociadas a sensores remotos se están desarrollando continuamente y permiten profundizar en los mecanismos funcionales de estos ecosistemas y ampliar la escala geográfica de las evaluaciones. La posibilidad de hacer estudios retrospectivos y las facilidades para la obtención de datos primarios de herramientas online como el Climate Engine permiten también la descripción de las tendencias y la evaluación de impactos previos, aunque su empleo no es aún muy frecuente (Pinheiro et al. 2019). Estudios de distribución, fragmentación, fenología y muchos otros se hacen posibles con la combinación de estas herramientas. El estudio de impactos en la vegetación generalmente ha estado limitado por la necesidad de hacer diseños de tipo BACI (Before - After - Control - Impact) (Underwood, 1992) para lograr una fortaleza estadística y metodológica suficiente, pero las potencialidades de las imágenes satelitales facilitan grandemente su aplicación.

\section{RESUMEN}

Introducción: Los manglares son una de las formaciones vegetales de mayor relevancia global por los servicios ecosistémicos y roles ecológicos que desempeñan, y representan el $5 \%$ de la superficie emergida de Cuba. No existen estudios previos que describan las variaciones en los índices espectrales de vegetación por sensores remotos en los manglares cubanos, pero con este tipo de variable se pueden constituir indicadores del estado de estos ecosistemas y emplearse en evaluaciones a nivel nacional. Objetivo: En el presente trabajo se evalúa la variabilidad espacial y temporal de dos índices espectrales de vegetación en cuatro manglares periurbanos de la ciudad de La Habana y se comparan con un sitio natural como control. Métodos: El estudio se desarrolló en las localidades de Bajo de Santa Ana, Cojímar, Rincón de Guanabo y la laguna El Cobre Itabo y el control fue tomado en la ciénaga de Zapata. A través de la plataforma Climate Engine se extrajeron, de 2 460 imágenes satelitales LandSat, los valores promedio de los índices espectrales NDVI y EVI en 5 parcelas por cada localidad de estudio, entre 1984 y 2019. Las variables se compararon estadísticamente entre localidades y se describió su tendencia a lo largo del periodo de estudio. Resultados: Se detectaron diferencias geográficas en los índices, que pueden correlacionarse con las propiedades estructurales y grado de conservación de cada localidad. La tendencia global de los índices fue a un aumento, pero difirió entre 
localidades. Los menores cambios se produjeron en el sitio control y, entre los humedales periurbanos, en el Rincón de Guanabo y en Cojímar. El ordenamiento de las localidades a partir de variables espectrales fue consistente con el de grado de conservación general. Conclusiones: La respuesta espectral permitió caracterizar cada manglar de manera individualizada, mostrando relación aparente con las características ecológicas y de conservación de cada lugar. Es necesario continuar promoviendo estudios con sensores remotos de estas formaciones boscosas y generar indicadores sólidos que sustenten su uso en futuras investigaciones y monitoreos en manglares del Caribe.

Palabras clave: humedales costeros, NDVI, EVI, indicadores ecológicos, tendencias históricas.

\section{REFERENCIAS}

Al Habshi, A., Youssef, T., Aizpuru, M., \& Blasco, F. (2007). New mangrove ecosystem data along the UAE coast using remote sensing. Aquatic Ecosystem Health and Management, 10(3), 309-319.

Alongi, D.M. (2002). Present state and future of the world's mangrove forests. Environmental Conservation, 29, 331-349.

Asner, G. (1998). Fuentes biofísicas y bioquímicas de variabilidad en la reflectancia del dosel. Teledetección del Medio Ambiente, 64, 234-253.

Boon, B., Zubir, M., Jafri, M., \& Hwee, L. (2011). Reflectance Characteristic of Certain Mangrove Species at Matang Mangrove Forest Reserve, Malaysia. Presentado en Proceeding of the 2011 IEEE International Conference on Space Science and Communication (IconSpace). Penang, Malaysia.

Cissell, J.M., \& Steinberg, J.R. (2018). Mapping forty years of mangrove cover trends and their implications for flats fisheries in Ciénaga de Zapata, Cuba. Environmental Biology of Fishes, Springer Nature. DOI: 10.1007/S10641-018-0809-0

Climate Engine (2019): [Software]. Recuperado de https:// app.climateengine.org/climateEngine

Huntington, J. L., Hegewisch, K.C., Daudert, B., Morton, C. G., Abatzoglou, J. T., Mcevoy, D. J., \& Erickson, T. (2017). Climate Engine. Cloud Computing and Visualization of Climate and Remote Sensing Data for Advanced Natural Resource Monitoring and Process Understanding. American Meteorological Society, BAMS, 2397-2409. DOI: 10.1175/ BAMS-D-15-00324.1

Ellison, A.M., \& Farnsworth, E.J. (1996). Anthropogenic disturbance of Caribbean mangrove ecosystems: past impacts, present needs, and future predictions. Biotropica, 28, 549-565.
Everitt, J.H., Yang, C., Summy, K.R., Judd, F.W., \& Davis, M.R. (2007). Evaluation of color-infrared photography and digital imagery to map black mangrove on the Texas Gulf Coast. Journal of Coastal Research, 23(1), 230-235.

Galford, G.L., Fernandez, M., Roman, J., Monasterolo, I., Ahamed, S., Fiske, G. ... Kaufman, L. (2018). Cuban land use and conservation, from rainforests to coral reefs. Bulletim of Marine Science, 94(2). DOI: 10.5343/bms.2017.1026

Gilman, E., Ellison, J., Duke, N.C., \& Field, C. (2008). Threats to mangroves from climate change and adaptation options: a review. Aquatic Botany, 89, 2, 237-250.

Giri, C., Ochieng, E., Tieszen, L.L., Zhu, Z., Singh, A., Loveland, T., Masek, J., \& Duke, N. (2011). Status and distribution of mangrove forests of the world using earth observation satellite data. Global Ecology and Biogeography, 20, 154-159.

Green, E.P., Clark, C., Mumby, P.J., Edwards, A. J., \& Ellis, A. C. (1998) Remote sensing techniques for mangrove mapping. International Journal of Remote Sensing, 19, 935-956.

Green, E.P., Mumby, P.J., Edwards, A. J., Clark, C.D., \& Ellis, A. C. (1997). Estimating leaf area index of mangroves from satellite data. Aquatic Botany, 58(1), 11-19.

Guzmán, J. M., \& Menéndez, L. (2013). Protocolo para el monitoreo del ecosistema de manglar. La Habana, Cuba: CNAP.

Hardisky, M.A., Gross, M.F., \& Klemas, V. (1986). Remote sensing of coastal wetlands. Bioscience, 36, 453-460.

Heumann, B.W. (2011). Satellite remote sensing of mangrove forests: recent advances and future opportunities. Progress in Physical Geography, 35(1), 87-108.

Huete, A. (1988). A soil-adjusted vegetation index (SAVI). Remote Sensing of Environment, 25, 295-309.

Huete, A., Didan, K., Miura, T., Rodriguez, E.P., Gao, X., \& Ferreira, L.G. (2002) Overview of the Radiometric and Biophysical Performance of the MODIS Vegetation Indices. Remote Sensing of Environment, 83, 195-213.

Jensen, J.R., Lin, H., Yang, X., Ramsey III, E., Davis, B.A., \& Thoemke, C.W. (1991). The measurement of mangrove characteristics in southwest Florida using SPOT multispectral data. Geocarto International, 6(2), 13-21

Kamaruzaman, J., \& Kasawani, I. (2007). Imaging spectrometry on mangrove species identification and mapping in Malaysia. Wseas Transactions on Biology and Biomedicine, 8(4), 118-126. 
Kovacs, J.M., Wang, J., \& Blanco-Correa, M. (2001). Mapping disturbance in a mangrove forest using multidate Landsat imagery. Environmental Management, 27(5), 763-776.

Krauss, K.W., Lovelock, C.E., McKee, K.L., López-Hoffman, L., Ewe, S.M., \& Sousa, W.P. (2008). Environmental drivers in mangrove establishment and early development: A review. Aquatic Botany, 89, 105-127.

Kuenzer, C., Bluemel, A., Gebhardt, S., Vo Quoc, T., \& Dech, S. (2011). Remote Sensing of Mangrove Ecosystems: A Review. Remote Sensing, 3, 878-928.

Menéndez, L., \& Guzmán, J.M. (Eds.) (2006). El ecosistema de manglar en el archipiélago cubano: estudios y experiencias enfocadas a su gestión. La Habana. Cuba: Editorial Academia.

Menéndez, L., Guzmán, J.M., \& Capote, R.T. (2002). Los manglares del archipiélago cubano: aspectos de su funcionamiento. En J.J. Neiff (Ed.), Humedales de Iberoamérica (pp. 237-251). Argentina: CYTED, Programa Iberoamericano de Ciencia y Tecnología para el Desarrollo.

Nagelkerken, I., Blaber, S.J.M., Bouillon, S., Green, P., Haywood, M., \& Kirton, L.G. (2008). The habitat function of mangroves for terrestrial and marine fauna: A review. Aquatic Botany, 89, 155-185.

Nayak, S., Anjali, B. \& Shah, D.G. (2003). Community zonation of selected mangrove habitat of India using satellite data. Scientific Note.

Pastor-Guzmán, J., Dasha, J., \& Atkinson, P.M. (2018) Remote sensing of mangrove forest phenology and its environmental drivers. Remote Sensing of Environment, 205, 71-84.

Roman, J. (2018). The ecology and conservation of Cuba's coastal and marine ecosystems. Bulletin of Marine Science, 94(2), 149-169.

Satyanarayana, B., Mohamad, K.A., Idris, I.F., Husain, M.L., \& Dahdouh-Guebas, F. (2011) Assessment of mangrove vegetation based on remote sensing and ground-truth measurements at Tumpat, Kelantan Delta, East Coast of Peninsular Malaysia. International Journal of Remote Sensing 32 (6), 1635-1650

Pinheiro, M.A.B., de Carvalho, M., da Rocha, J., Chaves, M.E.D., de Azevedo Silva, P.A., Alacoque, J.G., \& Trindade, F.S. (2019). Modelagem espectro-temporal do NDVI obtido de imagens Landsat 7 e 8 aplicado na cafeicultura. Presentado en Anais do XIX Simpòsio Brasileiro de Sensoriamento Remoto. INPE, Santos, Brasil.

Shah, G.D., \& Anjali, B. (2007). Mangrove spectral signatures: The need for hyper spectral. NRDMS-DST
Advanced Working Group Meet on Hyperspectral Signature Database Management System- A Development Perspective.

StatSoft, Inc. (2007). STATISTICA (data analysis software system) (version 8.0). Recuperado de www.statsoft. com

Suárez, R. (2011). Estado de salud de los ecosistemas de manglar de La Habana, Cuba (Tesis de pregrado). Universidad de La Habana, Cuba.

Thangaradjou, T., Sivakumar, K., Anantharaman, P., \& Kannan, L. (2007). Hyperspectral signatures for coastal and marine resources. Extended abstract volume of NRDMS-DST advanced working group meet on hyperspectral signature database management system- A development perspective, pp. 56-59.

Thomas, N., Bunting, P., Hardy, A., Lucas, R., Rosenqvist, A., \& Fatoyinbo, T. (2018). Mapping mangrove baseline and time-series change extent: A global monitoring approach. Remote Sensing, 10, 1466-1486.

Tong, P.H.S., Auda, Y., Populus, J., Aizpuru, M., Al-Habshi, A., \& Blasco, F. (2004). Assessment from space of mangrove evolution in the Mekong Delta, in relation to extensive shrimp farming. International Journal of Remote Sensing, 25, 4795-4812.

Underwood, A.J. (1992). Beyond BACI: the detection of environmental impacts on populations in the real, but variable, world. Journal of Experimental Marine Biology and Ecology, 161, 145-178.

Vaiphasa, C., Skidmore, A.K., \& Boer, de W.F. (2005). A post-classifier for mangrove mapping using ecological data. ISPRS Journal of Photogrammetry and Remote Sensing, 61(1), 1-10.

Valiela, I., Bowen, J.L., \& York, J.K. (2001). Mangrove forests: one of the world's threatened major tropical environments. BioScience, 51(10), 807-815.

Ventura, D., Fuente, A., Vicente, P., Isse, M., \& RodríguezOchoa, A. (2017). Evaluación cuantitativa de las afectaciones en la estructura de la vegetación en dos manglares de La Habana, Cuba. Informe de Trabajo Biológico de Campo II. Facultad de Biología, Universidad de La Habana, Cuba.

Wang, L., Sousa, W.P., Gong, P., \& Biging, G.S. (2004). Comparison of IKONOS and QuickBird images for mapping mangrove species on the Caribbean coast of Panama. Remote Sensing of Environment, 91(3), 432-440.

Xue, S., Hai-Shan, C., \& Zhi Hua, G. (2011). Remote Sensing of Mangrove Wetlands Identification. Procedia Environmental Sciences, 10, 2287-2293. 